\title{
Questioning The Fidelity Of The Next Generation Science Standards For Astronomy And Space Sciences Education
}

Stephanie J. Slater, Ph.D., CAPER Center for Astronomy \& Physics Education Research, USA

Timothy F. Slater, Ph.D., University of Wyoming, USA

\begin{abstract}
Although the Next Generation Science Standards (NGSS) are not federally mandated national standards or performance expectations for K-12 schools in the United States, they stand poised to become a de facto national science and education policy, as state governments, publishers of curriculum materials, and assessment providers across the country consider adopting them. In order to facilitate national buy-in and adoptions, Achieve, Inc., the non-profit corporation awarded the contract for writing the NGSS, has repeatedly asserted the development of the Standards to be a state-driven and transparent process, in which the scientific content is taken "verbatim", from the 2011 NRC report, Frameworks for K-12 Science Education: Practices, Crosscutting Concepts, and Core Ideas. This paper reports on an independently conducted fidelity check within the content domain of astronomy and the space sciences, conducted to determine the extent to which the NGSS science content is guided by the Frameworks, and the extent to which any changes have altered the scientific intent of that document. The side-by-side, two-document comparative analysis indicates that the science of the NGSS is significantly different from the Frameworks. Further, the alterations in the science represent a lack of fidelity, in that they have altered the parameters of the science and the instructional exposure (e.g., timing and emphasis). As a result the NGSS are now poised to interfere with widely desired science education reform and improvement. This unexpected finding affords scientists, educators, and professional societies with an opportunity, if not a professional obligation, to engage in positively impacting the quality of science education by conducting independent fidelity checks across other disciplines. This could provide a much needed formal support and guidance to schools, teachers, curriculum developers, and assessment providers.
\end{abstract}

Keywords: Astronomy Education; Education Standards; Performance Expectations; Next Generation Science Standards; NGSS

\section{INTRODUCTION}

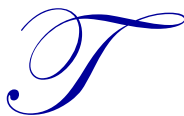

he Next Generation Science Standards (NGSS) are rapidly becoming a de facto national science education policy, as they hold promise to serve as a substantial improvement over previous science education reform efforts by the American Association for the Advancement of Science (AAAS, 1993), the National Research Council (NRC, 1996), and individual state's departments of education. Developed with warrant to the National Governors Association and drafted under the leadership of a non-profit corporation, Achieve, Inc. (2014) created and reportedly finalized the NGSS in April 2013 in a process that was reported to be rigorous and transparent, involving several drafts. According to Achieve, Inc., tens of thousands of public comments heavily influenced these drafts, including those of professional scientific societies, scientists, and educators (NGSS, 2013). Further, it was reported that a "fidelity check" was conducted (Robelen, 2013), reporting consistency between the guiding NRC (2011) report, Frameworks for K-12 Science Education: Practices, Crosscutting Concepts, and Core Ideas (Frameworks), and the final version of the NGSS. 
Concurrent with this activity, leading scientists across the scientific community have increasingly encouraged fellow scientists and professional societies to engage in supporting and improving science education classroom curriculum materials (Alberts, 2012; Coffey \& Alberts, 2013). While scientists are not always professional educators, professional scientists and their societies arguably serve as the best qualified to determine the emphases and accuracy of the science. As such, Achieve, Inc. has indicated that numerous scientists participated in the drafting of the NGSS scientific content. Well-respected, prominent scientists engaged in the drafting of the Frameworks, and in the process of creating NGSS drafts, participating scientists (including the authors) provided careful feedback. However, the extended duration of the NGSS development process, the large number of drafts involved, and a lack of continuity in the science teams involved in each stage of the process resulted in a situation in which most scientists only provided one-time feedback on only one of the NGSS versions. There were few, if any, scientists in place to provide corporate memory. As a result, it is possible that many in the scientific community, including professional scientific societies, have not paid sufficient attention to the recently released NGSS drafts, putting faith in the perceived transparency of the process and the assurances that the NGSS would follow the scope and sequence of the Frameworks. Indeed, several professional societies endorsed the NGSS prior to the release of the final draft, and none of the professional societies reviewed versions of the NGSS that were released subsequent to the "final" April 2013 version. Given that endorsements and statements from professional societies would likely be used to support the broad implementation of the NGSS and could be used to influence political and funding entities, such societies have an ethical responsibility to engage in due diligence and to conduct an independent, systematic analysis of the finalized documents prior to making a statement of support or endorsement.

Content provided by Achieve, Inc. give reason to believe that due diligence may be necessary. The drafting of the NGSS involved three public reviews of three NGSS drafts, with alterations occurring to the NGSS content at every stage. At the same time the bottom of every page of the NGSS includes a statement that asserts that the science is "reproduced verbatim" from the Frameworks (Figure 1).

\footnotetext{
*The performance expectations marked with an asterisk integrate traditional science content with engineering through a Practice or Disciplinary Core Idea. The section entitled "Disciplinary Core Ideas" is reproduced verbatim from A Framework for K-12 Science Education: Practices, Cross-Cutting Concepts, and Core Ideas. Integrated November 2013 and reprinted with permission from the National Academy of Sciences. C2013 Achieve, Inc. All rights reserved. 5 of 103
}

Figure 1. Statement of "verbatim" language from the NGSS.

It is difficult to conceive of any pathway in which the stakeholder feedback could be incorporated, while at the same time still retaining the precise, unaltered language of the Frameworks across three non-identical public drafts. Potentially, Achieve, Inc. could have incorporated feedback into versions of the NGSS, providing a nonverbatim version of the Frameworks science; or, Achieve, Inc. could have preserved the unaltered text of the Frameworks without incorporating feedback. It cannot be that both conditions are true; by definition, one cannot edit text while maintaining that text in an unedited format.

A cursory search through the NGSS and the Frameworks indicates that the science of the NGSS is not verbatim from the Frameworks. For instance, the "final" NGSS version from April 9, 2013 (p. 53), hereafter called NGSS-April, we noted that middle school standard "PS3.A" stated:

Temperature is not a measure of energy. The relationship between the temperature and the total energy of a system depends on the types, states, and amounts of matter present [emphasis added].

The corresponding PS3.A in the Frameworks, we found that the text is nearly identical, with the exception of the word "not" in the passage. In other words, in moving from the Frameworks to the NGSS, text was added in such a way that the NGSS argued for science that is diametrically opposed to that given in the Frameworks.

A subsequent "final" version of the NGSS was uploaded on the Achieve, Inc. website more than a month after the final NGSS were released. In this "May 2013 final version" of the NGSS, the text describing the science of temperature was altered: 
The temperature of a system is proportional to the average internal kinetic energy and potential energy per atom or molecule (whichever is the appropriate building block for the system's material). The details of that relationship depend on the type of atom or molecule and the interactions among the atoms in the material. Temperature is not a direct measure of a system's total thermal energy. The total thermal energy (sometimes called the total internal energy) of a system depends jointly on the temperature, the total number of atoms in the system, and the state of the material. (pp. 54-55)

The source of this new definition of temperature remains unknown, and to our knowledge, this standard has not been subject to public review or comment. However, it remains clear that this did not come verbatim from the Frameworks, for no such description of temperature appears there. Further, this is a substantive alteration of the scope of the science to be taught at this grade band, and involves a topic that is important in all branches of science and engineering. As such, this alteration to the text represents a lack of fidelity between the NGSS and the Frameworks. This provides sufficient warrant to conduct an independent analysis of the fidelity of the science contained in the NGSS.

While pundits across the spectrum have engaged in ongoing discussions related to the appropriateness of the NGSS based upon educational priorities, depth, breadth, politics, and religion, this paper concerns a far more straightforward matter: Do the NGSS demonstrate fidelity with the vision of the science content and disciplinary structure presented in the Frameworks? Accordingly, this paper addresses two questions: 1) Does the scientific content of the NGSS show fidelity to the scientific content of the Frameworks within the domain of astronomy and the space sciences; and 2) If the NGSS demonstrates variation from the science of the Frameworks within this content domain, is the alteration scientifically substantive? This paper briefly describes the methods and results of an independent fidelity check process, provides examples of the findings, and finally, makes some recommendations for further steps.

\section{METHOD}

\section{Defining "Fidelity"}

The fidelity check reported by the NRC and Achieve, Inc. has not been publically described, necessitating the construction of a definition of fidelity, and a process by which to determine fidelity. Relevant definitions from a standard dictionary provide that fidelity is an adherence to facts, details, accuracy, and exactness. In educational settings, fidelity also includes the concept of exposure, in which the timing and length of educational experiences adhere to original intentions, as they represent learning opportunities related to a portion of content. Our metrics followed from those qualifications. Given these definitions, fidelity between the science of the NGSS and the Frameworks is based on the following conditions:

The science in the NGSS must faithfully adhere to the science in the Frameworks, in detail and accuracy.

$\circ \quad$ Subtractions from or additions to the substance of the science constitute a lack of fidelity.

- Substantive changes to important parameters of the science constitute a lack of fidelity.

The science in the NGSS must faithfully adhere to exposure of the science as delineated in the Frameworks.

$\circ \quad$ Alterations in the timing of the scientific content (e.g., gradeband) constitute a lack of fidelity.

$\circ \quad$ Alterations in the emphasis of the science represent a lack of fidelity.

Finally, the NGSS claimed that some states acknowledge the "performance expectations" as the functional standards and that "all students should be held accountable for demonstrating their achievement of all performance expectations." Therefore, we included the science content of the performance expectations (PE) in our analysis.

While learning science is more than about learning facts, other aspects of a fidelity check are set aside for another study. While cross-cutting concepts and disciplinary practices are crucial to the process of enculturating students into science, the science content represents the substrate upon which students practice these more complex skills. As such, making sure that the science is accurate and as intended is a barrier that must be crossed before those other conversations can become meaningful. 


\section{Data Sources}

We judged the entire NGSS document itself as too large for a single, comprehensive review across all discliplines. Moreover, scientific and discipline-based education researchers may be best poised to do the analyses of the content domains within their own fields of expertise. Given the authors' expertise, this study is constrained to an analysis of the portions of the NGSS Disciplinary Core Ideas (DCI) most closely related to astronomy and planetary sciences. NRC's Frameworks served as the primary data source because the NGSS are a purported derivative. We then determined which version of the NGSS to use for a comparison document. While the official "final" NGSS were released on April 9, 2013, further investigation indicates that other "final" drafts were released again in May and then in June, July, August and November of 2013. (Given that these releases were unannounced, it is possible that there are additional, unknown releases.) Each document contains changes to the science. However, the November 2013 is the versions posted online at the time of this writing, and serves as the data source for comparisons to the Frameworks.

\section{Understanding the Documents}

Although suggested as being a highly difficult to interpret technical document (Robelen, 2013), the NGSS are readily accessible. The science content targeted by this analysis, the DCIs, can be found in the middle of each page in orange colored boxes. The corresponding performance assessments (PEs) are found in the white box at the top of each table as shown in Figure 2.

In the NGSS, the science content is coded with a series of letters and numbers. For instance, content required for a coherent understanding of astronomy and the space sciences can be found across the Earth and Space Science and Physical Science domains. These domains are coded as "ESS" and "PS", respectively. The Earth and Space Sciences are divided into three parts, which are coded "ESS1," "ESS2," and "ESS3." Each of these constructs is divided into small concepts, which are also coded. For example, ESS1, "Earth's place in the universe," is broken down into three parts: "ESS1.A: The Universe and Its Stars," "ESS1.B: Earth and the Solar System," and "ESS1.C: The History of Planet Earth." This system, derived from the Frameworks, is used consistently throughout both documents, allowing for easy comparison of the scientific content between the two documents. 


\begin{tabular}{|c|c|c|c|}
\hline \multicolumn{4}{|c|}{$\begin{array}{l}\text { Next Generation Science Standards } \\
\text { 5. Earth's Systems }\end{array}$} \\
\hline \multicolumn{4}{|c|}{ Students who demonstrate understanding can: } \\
\hline 5-ESS2-1. & \multicolumn{3}{|c|}{$\begin{array}{l}\text { Develop a model using an example to describe ways the geosphere, biosphere, hydrosphere, and/or } \\
\text { atmosphere interact. [Clarification Statement: Examples could include the influence of the ocean on } \\
\text { ecosystems, landform shape, and climate; the influence of the atmosphere on landforms and ecosystems } \\
\text { through weather and climate; and the influence of mountain ranges on winds and clouds in the atmosphere. } \\
\text { The geosphere, hydrosphere, atmosphere, and biosphere are each a system.] [Assessment Boundary: } \\
\text { Assessment is limited to the interactions of two systems at a time.] }\end{array}$} \\
\hline 5-ESS2-2. & \multicolumn{3}{|c|}{$\begin{array}{l}\text { Describe and graph the amounts and percentages of water and fresh water in various reservoirs to } \\
\text { provide evidence about the distribution of water on Earth. [Assessment Boundary: Assessment is limited } \\
\text { to oceans, lakes, rivers, glaciers, ground water, and polar ice caps, and does not include the atmosphere.] }\end{array}$} \\
\hline 5-ESS3-1. & \multicolumn{3}{|c|}{$\begin{array}{l}\text { Obtain and combine information about ways individual communities use science ideas to protect the } \\
\text { Earth's resources and environment. }\end{array}$} \\
\hline \multicolumn{4}{|c|}{$\begin{array}{l}\text { The performance expectations above were developed using the following elements from the NRC document } A \text { Framework for } \\
K-12 \text { Science Education: }\end{array}$} \\
\hline \multicolumn{2}{|c|}{$\begin{array}{l}\text { Science and Engineering Practices } \\
\text { Developing and Using Models } \\
\text { Modeling in 3-5 builds on K-2 } \\
\text { experiences and progresses to building } \\
\text { and revising simple models and using } \\
\text { models to represent events and design } \\
\text { solutions. } \\
\text { - Develop a model using an example } \\
\quad \text { to describe a scientific principle. } \\
\quad \text { (5-ESS2-1) } \\
\text { Using Mathematics and } \\
\text { Computational Thinking } \\
\text { Mathematical and computational } \\
\text { thinking in 3-5 builds on K-2 } \\
\text { experiences and progresses to extending } \\
\text { quantitative measurements to a variety } \\
\text { of physical properties and using } \\
\text { computation and mathematics to } \\
\text { analyze data and compare alternative } \\
\text { design solutions. } \\
\text { Describe and graph quantities such } \\
\text { as area and volume to address } \\
\text { scientific questions. (5-ESS2-2) } \\
\text { Obtaining, Evaluating, and } \\
\text { Communicating Information } \\
\text { Obtaining, evaluating, and } \\
\text { communicating information in } 3-5 \\
\text { builds on K-2 experiences and } \\
\text { progresses to evaluating the merit and } \\
\text { accuracy of ideas and methods. } \\
\text { Obtain and combine information } \\
\text { from books and/or other reliable } \\
\text { media to explain phenomena or } \\
\text { solutions to a design problem. (5- } \\
\quad \text { ESS3-1) }\end{array}$} & $\begin{array}{l}\text { ESS2.A: Earth Materials and } \\
\text { Systems } \\
\text { Earth's major systems are the } \\
\text { geosphere (solid and molten rock, } \\
\text { soil, and sediments), the } \\
\text { hydrosphere (water and ice), the } \\
\text { atmosphere (air), and the biosphere } \\
\text { (living things, including humans). } \\
\text { These systems interact in multiple } \\
\text { ways to affect Earth's surface } \\
\text { materials and processes. The ocean } \\
\text { supports a variety of ecosystems } \\
\text { and organisms, shapes landforms, } \\
\text { and influences climate. Winds and } \\
\text { clouds in the atmosphere interact } \\
\text { with the landforms to determine } \\
\text { patterns of weather. (5-ESS2-1) } \\
\text { ESS2.C: The Roles of Water in } \\
\text { Earth's Surface Processes } \\
\text { Nearly all of Earth's available } \\
\text { water is in the ocean. Most fresh } \\
\text { water is in glaciers or underground; } \\
\text { only a tiny fraction is in streams, } \\
\text { lakes, wetlands, and the } \\
\text { atmosphere. (5-ESS2-2) } \\
\text { ESS3.C: Human Impacts on Earth } \\
\text { Systems } \\
\text { Human activities in agriculture, } \\
\text { industry, and everyday life have } \\
\text { had major effects on the land, } \\
\text { vegetation, streams, ocean, air, and } \\
\text { even outer space. But individuals } \\
\text { and communities are doing things } \\
\text { to help protect Earth's resources } \\
\text { and environments. (5-ESS3-1) }\end{array}$ & $\begin{array}{l}\quad \text { Crosscutting Concepts } \\
\text { Scale, Proportion, and Quantity } \\
\text { Standard units are used to measure and } \\
\text { describe physical quantities such as } \\
\text { weight and volume. (5-ESS2-2) } \\
\text { Systems and System Models } \\
\text { A system can be described in terms of } \\
\text { its components and their interactions. } \\
\text { (5-ESS2-1),(5-ESS3-1) } \\
\text { Connections to Nature of Science } \\
\text { Science Addresses Questions About } \\
\text { the Natural and Material World. } \\
\text { - Science findings are limited to } \\
\text { questions that can be answered } \\
\text { with empirical evidence. (5-ESS3- } \\
\text { 1) }\end{array}$ \\
\hline
\end{tabular}

Figure 2. Next Generation Science Standards - Earth's Systems

(reproduced from http://www.nextgenscience.org/5es-earths-systems) 


\section{A Simple Fidelity Check Process}

The work of conducting a fidelity check is a remarkably simple process. The process used in this study required very little time, very few people, and no funding. Two people (the authors) conducted the fidelity review in a few hours with downloaded PDF versions of the Frameworks and the NGSS. (We used the NGSS formatted as "Combined DCIs"). The documents were projected onto an office wall to expedite the work and facilitate discussion, but this was a choice of convenience rather than of necessity.

First, the Frameworks were consulted in order to determine the location of science content relevant to the analysis. Given that astronomy and the Earth and Space Sciences are interdisciplinary fields, this review likely required more time than would be needed for the more pure sciences. However, even in the case of an interdisciplinary science, the table of contents in the Frameworks still streamlines much of the work. In addition to the whole of the Earth and Space Sciences content, content related to the Physical Sciences was included in this analysis. We recorded the codes for the relevant content in order to facilitate finding the material in both documents.

Beginning with the Standards that should be covered by the end of Grade 2, the NGSS and the Frameworks were compared side-by-side. Organized in a more coherent fashion, The Frameworks gives all of the content for a single code in one location, serving as a more stable reference point. The NGSS is organized in a different fashion, with single codes that appear over several different pages, meaning the content is described on sometimes widely separated pages. However, it is not necessary to scroll through all of the pages to find the content of interest, as most PDF readers have a search function. By entering the content code (e.g., "ESS1.A") into the search function, it is possible to rapidly find all references to that content within the Pre-K through Grade 2 band (hereafter called the "Grade 2 band"). Using this method, it is a simple matter to engage in a process of comparative inspection to determine whether or not discrepant changes existed to ESS1.A in the Grade 2 band, in only a few minutes. In this process, if both readers agree that scientific content had altered, the section code, grade band, and content alterations is recorded for subsequent analysis. For this study, this process required approximately two hours, employing two knowledgeable researchers. Larger teams could divide targeted content and likely finish this process in less time.

After compiling a list of alterations to the science, the authors calculated the percentage of text that had been altered and the distribution of the alterations across the content, as an extremely gross measure of the amount of text that had been altered. This measure is not a clear measure of a lack of fidelity, requiring each alteration to be categorized, depending upon the nature of the change. Given the study's working definition of fidelity, the categorized changes were used to determine if the science content displays fidelity in terms of scientific parameters, sequencing in the grade bands, and depth of coverage. The changes in the text were therefore classified into the following categories:

- Typos/grammar/syntax: These changes reflect an attempt to improve the communication of the science content by correcting errors of writing.

- Superficial abbreviations: These changes decrease the number of words required to convey meaning, particularly in explicating examples, but do not function to change the science content of the text. For example, in PS1.A, the Frameworks provides the following text: "Different kinds of matter exist (e.g., wood, metal, water), and many of them can be either solid or liquid, depending on temperature." The NGSS deletes the parenthetical "e.g., wood, metal, water." In this case, the deleted words were not deemed necessary to specify or define parameters for the content. Those words could easily be replaced with countless other equivalent word choices without changing the intended content ("e.g., glass, wax, salts").

- Substantive abbreviations: These changes decrease the number of words required, particularly in explicating examples, and do so in such a way that remaining text no longer reflects the Frameworks' intended specificity. More to the point, such changes alter the implied parameters for instruction. For example, in ESS1.B, the Frameworks provides the following text:

The orbits of Earth around the sun and of the moon around Earth, together with the rotation of Earth about an axis between its North and South poles, cause observable change. These include day and night; daily and seasonal changes in the length and direction of shadow; phases of the moon; and different positions of the sun, moon and stars at different times of the day, month, and year. 
The NGSS deletes the words "seasonal changes" and "phases of the moon." In this example, the Frameworks provide explication of the specific changes that should be understood at this level, parameterizing the scientific content. By selectively deleting two of the specific examples, the scope of the intended scientific content has been changed. In the authors' expert judgment, founded on decades of experience writing curricula that support science standards, this change to the wording will impact future curriculum development and subsequent instruction and assessment in classrooms, and is therefore a substantive alteration.

- Changes in the timing of instruction: These changes involve science content that is moved from its specified grade band placement in the Frameworks to another grade band in the NGSS. These changes reflect a lack of fidelity in that they are not aligned with the correct developmental age of instruction, as determined by the expert writers of the Frameworks. These writing teams involved not only traditional scientists, but psychologists and cognitive scientists who study child development and its interaction with a child's readiness to learn. For example, in PS4.A, the Frameworks provide the following text at the Grade 2 band:

Waves, which are regular patterns of motion, can be made in water by disturbing the surface. When waves move across the surface of deep water, the water goes up and down in place; it does not move in the direction of the wave - observe, for example, a bobbing cork or seabird - except when the water meets the beach. Sound can make matter vibrate, and vibrating matter can make sound."

The NGSS moves this content, with the exception of the last sentence, to the Grade 5 band. As such, the order of instruction now requires mastery of waves that cannot be seen (sound) through matter that cannot be seen (air) prior to mastery of waves that can be observed through matter that can be seen, felt, and often heard. As such, this alteration in sequence likely diminishes students' ability to master the wave concept, providing a good example of the ways in which a change in timing of instruction constitutes a lack of fidelity with educational implications.

- Deletion of concepts: These changes involve deletions of content that go beyond an abbreviation of text. Rather, these changes involve the deletion of whole scientific ideas. Some of the deletions may represent removal of content that the NGSS writers believed to be repetitions of content found elsewhere in the text. However, repetitions in content may be intended by the writers of the Frameworks, serving two educational purposes. The first is that repetition of content provides for a longer period of exposure to an idea, a common teaching technique for ensuring that students come to a coherent understanding of a difficult topic. For instance, the concept of evolution is one of the most important and one of the most ideas fundamental to understand in the sciences. As such, it should appear in many instances across the standards. The second function of repetition is to provide students with interdisciplinary educational experiences. Again using the example evolution, this concept is important in many fields beyond biology, including the Earth Sciences. In reading the Frameworks, one can see that the authors peppered the standards with ideas related to evolution, and it is emphasized in the Earth Sciences, particularly in section ESS2.E. The NGSS removes the majority of that content. These purposeful omissions represent a substantive alteration to the intent of the Frameworks.

The first two categories of changes ("Typos/grammar/syntax" and "Superficial abbreviations") are not considered to impact the fidelity of the NGSS in a meaningful way. The last three categories of alterations ("Substantive abbreviations", "Changes in the timing of instruction", and "Deletion of concepts") are considered to alter the intent, sequence, or depth of coverage of the content in such a way that they reduce the fidelity of the $N G S S$. 


\section{RESULTS}

For this paper, fidelity was considered related to the science contained in the following sections of the Frameworks shown in Figure 3:

\begin{tabular}{|l|}
\hline \multicolumn{1}{|c|}{ Core Idea ESS1: Earth's Place in the Universe } \\
\hline ESS1.A: The Universe and Its Stars \\
\hline ESS1.B: Earth and the Solar System \\
\hline ESS1.C: The History of Planet Earth \\
\hline \\
\hline ESS2.A: Earth Materials and Systems Core Idea ESS2: Earth's Systems \\
\hline ESS2.B: Plate Tectonics and Large-Scale System Interactions \\
\hline ESS2.C: The Roles of Water in Earth's Surface Processes \\
\hline ESS2.D: Weather and Climate \\
\hline ESS2.E: Biogeology \\
\hline \\
\hline ESS3.A: Natural Resources \\
\hline ESS3.B: Natural Hazards \\
\hline ESS3.C: Human Impacts on Earth Systems \\
\hline ESS3.D: Global Climate Change \\
\hline
\end{tabular}

Figure 3. ESSE Standards reviewed.

In addition, fidelity was considered as it relates to some of the some material in the physical sciences, as an understanding of this material undergirds a coherent understanding of the science in astronomy and the Earth and Space Sciences. That science was contained in the following sections of the Frameworks shown in Figure 4:

PS1.A: Structure and Properties of Matter

Core Idea PS1: Matter and Its Interactions

PS1.B: Chemical Reactions

PS1.C: Nuclear Processes

PS2.A: Forces and Motion

Core Idea PS2: Motion and Stability: Forces and Interactions

PS2.B: Types of Interactions

PS2.C: Stability and Instability in Physical Systems

PS3.A: Definitions of Energy

PS3.B: Conservation of Energy and Energy Transfer

PS3.C: Relationship Between Energy and Forces

PS3.D: Energy in Chemical Processes and Everyday Life

PS4.A: Wave Properties

Core Idea PS4: Waves and Their Applications in Technologies for Information Transfer

PS4.B: Electromagnetic Radiation

PS4.C: Information Technologies and Instrumentation

Figure 4. Additional PS Standards reviewed.

\section{Gross Measures of Change}

For the entirety of the text, a simple word count comparison was completed in order to obtain a gross estimation of the degree to which the scientific content was altered. The listed sections of the Frameworks consist of 9,169 words, while the same sections of the NGSS consist of 5,559 words. By this measure, $40 \%$ of the scientific content text of the Frameworks was removed from the NGSS. 
In order to obtain a gross measure of the distribution of the alterations, a calculation was made of the percentage of sections that had been altered. Of the 25 sections of the science analyzed from the Frameworks (as described in Figures 3 and 4), one-hundred percent of these sections were altered to some extent. These sections are further divided into "grade bands," delineating when the science should be taught. The grade bands are set at the 2nd, 5th, 8th, and 12th grades. (For some science sections, certain grade bands were intentionally left blank in the Frameworks, indicating that the particular topic should not be taught within the given grade band.) The content of interest in this study is contained in 97 grade band benchmarks. Of those, 76 grade band benchmarks (78\%) were altered.

\section{Classifying the Nature of the Changes to the Science}

While gross measures of change prove that the science of the NGSS is not verbatim to the Frameworks as asserted by Achieve, Inc., it lacks sufficient evidence to determine whether or not the science passes a fidelity check. In order to address that question, we evaluated whether or not those changes are substantive, or do the changes alter the scientific meaning of the text or the sequence or scope of instruction? The following table illustrates the distribution of the 141 alterations made to the science content across the categories described in the previous sections. Figure 1 provides examples, raw number counts, and the percentage of total alterations.

Table 1. Characterization of Alterations of the Science in the NGSS

\begin{tabular}{|c|c|c|}
\hline CATEGORY & FREQUENCY & PERCENTAGE \\
\hline Category: Typos/grammar/syntax & $\begin{array}{l}\text { Number of instances: } \\
0 \text { (out of } 141 \text { changes) }\end{array}$ & $\begin{array}{l}\text { Percentage of all alterations } \\
0 \%\end{array}$ \\
\hline $\begin{array}{l}\text { Category: } \\
\text { Superficial abbreviations }\end{array}$ & $\begin{array}{l}\text { Number of instances: } \\
26 \text { (out of } 141 \text { changes) }\end{array}$ & $\begin{array}{l}\text { Percentage of all alterations } \\
18 \%\end{array}$ \\
\hline \multicolumn{3}{|c|}{$\begin{array}{l}\text { PS1.A: "All substances are made from some } 100 \text { different types of atoms, which combine with one another in various ways." } \\
\text { ESS1.C: "Some events, like an earthquake, happen very quickly; others, such as the formation of the Grand Canyon, occur } \\
\text { very slowly, over a time period much longer than one can observe" } \\
\text { ESS3.A: "Humans use natural resources for everything that they do: for example, they use soil and water to grow food wood } \\
\text { to burn to provide heat or to build shelters, and materials such as iron or copper extracted from Earth to make } \\
\text { cooking pans." }\end{array}$} \\
\hline $\begin{array}{l}\text { Category: } \\
\text { Substantive abbreviations }\end{array}$ & $\begin{array}{l}\text { Number of instances: } \\
5 \text { (out of } 141 \text { changes) }\end{array}$ & $\begin{array}{l}\text { Percentage of all alterations } \\
4 \%\end{array}$ \\
\hline
\end{tabular}

Examples of this type of change (deleted words shown in italics):

PS2.B: "Objects in contact exert forces on each other (friction, elastic pushes and pulls)."

PS2.B: "Electric, magnetic, and gravitational forces between a pair of objects do not require that the objects be in contact."

PS3.B: "Light also transfers energy from place to place. For example, energy radiated from the sun is transferred to Earth by light. When this light is absorbed, it warms Earth's land, air, and water and facilitates plant growth."

PS3.B: "Energy is transferred out of hotter regions of objects' and into colder ones by the processes of conduction, convection, and radiation."

\begin{tabular}{lll}
\hline Category: & Number of instances: & Percentage of all alterations \\
Changes in the timing of instruction & 3 (out of 141 changes) & $2 \%$ \\
\hline
\end{tabular}

Examples of this type of change (deleted words shown in italics):

All such changes are found in PS4.A. In this example, water waves are moved from Grade 2 band to Grade 5 band; wave interaction is moved from Grade 5 band to Grade 12 band; and the use of waves to determine the Earth's interior is moved from Grade 8 band to Grade 12 band. 
$\underline{\text { (Table } 1 \text { continued) }}$

\begin{tabular}{lll}
\multicolumn{1}{c}{ CATEGORY } & \multicolumn{1}{c}{ FREQUENCY } & \multicolumn{1}{c}{ PERCENTAGE } \\
\hline Category: & Number of instances: & Percentage of all alterations \\
Deletion of concepts & $\mathbf{1 0 7}$ (out of 141 changes) & $\mathbf{7 6 \%}$ \\
\hline
\end{tabular}

Examples of this type of change (deleted words shown in italics):

PS1.C: "Nuclear fusion taking place in the cores of stars provides the energy released (as light) from those stars and produced all of the more massive atoms from primordial hydrogen. Thus, [sic] the elements found on Earth and throughout the universe (other than hydrogen and most of helium, which are primordial) were formed in the stars or supernovas by fusion processes."

PS2.A: "In any system, total momentum is always conserved."

PS4.A: "Earthquakes cause seismic waves, which are waves of motion in Earth's crust."

PS4.B: "All electromagnetic radiation travels through a vacuum at the same speed, called the speed of light. Its speed in any other medium depends on its wavelength and the properties of that medium..."

ESS1.A: "The universe began with a period of extreme and rapid expansion known as the Big Bang."

ESS1.A: "The sun is just one of more than 200 billion stars in the Milky Way galaxy, and the Milky Way galaxy is just one of hundreds of billions of galaxies in the universe."

ESS1.C: "Radioactive decay lifetimes and isotopic content in rocks provide a way of dating rock formations and thereby fixing the scale of geological time."

ESS2.B: "Plate tectonics is the unifying theory that explains the past and current movements of the rocks at Earth's surface and provides a framework for understanding its geological history. Plate movements are responsible for most continental and ocean floor features and for the distribution of most rocks and minerals within Earth's crust."

ESS2.C: "Water is found almost everywhere on Earth: as vapor; as fog or clouds in the atmosphere; as rain or snow falling from clouds; as ice, snow, and running water on land and in the ocean; and as groundwater beneath the surface. The downhill movement of water as it flows to the ocean shapes the appearance of the land."

ESS2.D: "Greenhouse gases in the atmosphere absorb and retain the energy radiated from land and ocean surfaces, thereby regulating Earth's average surface temperature and keeping it habitable."

ESS2.D: "Climate change can occur when certain parts of Earth's systems are altered. Geological evidence indicates that past climate changes were either sudden changes caused by alterations in the atmosphere; longer term changes (e.g., ice ages) due to variations in solar output, Earth's orbit, or the orientation of its axis."

\section{DISCUSSION}

\section{Analyzing the NGSS DCIs for Core Ideas}

Specific scientific errors uncovered in analyzing the NGSS have been formally described elsewhere (Gross, 2011) and exceed the scope of this paper. However, it remains clear from this data that the NGSS fails to pass a cursory fidelity check, and it fails to pass all three criteria of fidelity. The $N G S S$ documents demonstrate a widespread and non-trivial deviation from the science content put forth in the Frameworks. Within the NGSS, the order in which content is intended to be taught has been rearranged, and the specific parameters defining the scientific content have often been removed. The NGSS has dramatically altered the length of time that concepts were intended to be taught, and finally, concepts have been deleted at multiple levels.

For scientists, educators, and administrators, this last finding should inspire the most concern. In the process of compiling the scope of changes made to the science, it was noticed that numerous scientific concepts have been deleted, entirely. This led to a search for the standards related to fundamental and significant concepts. Specific checks were conduced realted to the physical laws, the major theories of the science (e.g., Big Bang, plate tectonics), forces, light and scale, and structure of the universe. The documents were searched to determine if these concepts had been removed in instances, or if they had been removed from the scientific content entirely.

This secondary analysis led to a disturbing and important conclusion about the impact of these alterations: much of the scientific content deleted is fundamental in nature, and the widespread deletions are often literally or functionally comprehensive. As one example of a "functionally complete deletion," in referencing the Big Bang, the Frameworks introduced the concept at the middle school level by stating: "The universe began with a period of extreme and rapid expansion known as the Big Bang." The Frameworks' description of the core idea also indicates that the Big Bang occurred about 13.7 billion years ago. This concept is reinforced at the high school level with greater emphasis on the science of the evidence in the Frameworks. In stark contrast, the NGSS removes the topic of 
the Big Bang and a description of the Big Bang from the middle school level. The NGSS now lacks any indication that the universe began with the Big Bang or that the universe underwent a period of expansion. No mention of the age of the universe occurs. All that remain of the scientific concept of the Big Bang, in the NGSS, are high schoolband vague references to evidence, and that portion is rather bizarre; for instance, it refers to "non-stellar gases," a term that cannot be found outside of the NGSS. This represents a substantive change in the content, timing and duration of instruction on this topic. Moreover, very few students take a course that includes the topic of the Big Bang at the high school level, and as a consequence, most students educated in a system that is driven by the NGSS, will have little opportunity to encounter this content in their K-12 education. This represents a significant deviation from the intent of the scientific and education experts who authored the Frameworks, in terms of the science content, the timing of the content, and the number of exposures.

Other major concepts experienced the same sort of deletion from the content. The theory of plate tectonics, like the Big Bang theory, was removed from the middle school grades and only appears in the high school grades in terms of evidence for, or consequences of, tectonic activity. The NGSS content describing the scale and structure of the universe (the structure of the solar system, galaxy, and universe) is now missing. All reference to the evidence of evolution through the rock record has been deleted from the Earth Science standards.

Scientific content related to core physical laws has been removed entirely. For instance, the concept of the frictional force is central to the understanding of Newton's Laws. As alluded to earlier, the concept of "friction" appears in the Frameworks nine times as a grade-band endpoint: The concept of friction does not even appear once in the NGSS. The word is no longer in the document, with the exception of one appearance in the Performance Assessment for PS2.2, in which the document states: "Assessment does not include friction as a mechanism for change in speed." The concept of gravity appears, as a physical science topic, thirty-one times in the Frameworks, yet only seven times in the NGSS. While not deleted entirely, gravitational forces (but not electrical or magnetic forces) have effectively been stripped from the content. The same is true for mass-equivalence, optics, nearly every reference to Kepler's Laws and Newton's Universal Gravitation, conduction and convection, the water cycle, absolute dating, the concept of a pure substance, and the conservation of momentum. This is a non-exhaustive list.

Therefore, there is sufficient data to state that the fundamentals of the targeted science have been substantively, and negatively altered in the translation from the Frameworks to the NGSS. This data reveal a substantive and unacceptable lack of fidelity between the NGSS and the Frameworks that merits concern and caution by stakeholders.

\section{IMPLICATIONS}

The down-range implications of fundamental and unadvertised changes in the science of the Frameworks are alarming. Given these findings, other disciplinary experts are encouraged to conduct independent fidelity checks. Clearly the NGSS development process, despite its promise for fidelity, transparency, and rigor, was not able to fruitfully produce standards that remain faithful to the Frameworks or that meet a minimum requirement of scientific accuracy. As cited earlier, Achieve, Inc. documents vaguely report receiving more than 20,000 pieces of feedback, including comments from many leading scientific professional societies on its website. At least some of those received public comments arguably contained feedback that would have rectified the errors and devastating deletions that we have uncovered. It is reasonable to assert that if any attention had been paid to the feedback from stakeholders, at the very least, typographical errors would have been fixed. However, we cannot know definitively since the specific scientific feedback on earlier drafts of the NGSS is not available for inspection.

These findings are potentially disturbing for members of endorsing societies and for the numerous teachers and science education professionals who diligently provided feedback to the NGSS drafts. In effect, these groups provided "expert warrant" for the NGSS, a claim prominently displayed on the NGSS website. These endorsements are intended to demonstrate widespread scientific support and implied ratification of the NGSS and certainly are intended to encourage adoption of the NGSS in states across the nation. Such statements hold power since they are perceived as published assertions that the NGSS reflects essential scientific content for all K-12 students to learn. By extension, one can very reasonably consider excluded ideas as less important. 
As a consequence, such statements present potential harm. In the debate about the NGSS, supporters have asserted that flaws in the NGSS can be fixed at the local level, rendering missteps in the $N G S S^{\prime}$ scientific content moot. These assertions are both odd and pragmatically unrealistic. It makes little sense to put forth a document that details the core science education every American student should learn without validating the accuracy and coherence of the science. At the same time, the weight of the societal endorsements make changing the standards very difficult for any who would like to repair the content, as they will have an enormous structure to battle. When state-level Departments of Education, publishers of curriculum materials, and outreach professionals begin to implement these standards, they will be expected to act on the NGSS rather than on the Frameworks. If the Big Bang does not appear in the middle school bands of the NGSS, then it will unlikely be written into middle school course descriptions, outlines, assessments, or textbooks. If friction does not appear in the NGSS, it will be squeezed out of the lab manuals and the course syllabus. If educational professionals believe that the Big Bang theory should be taught in our schools, they will have to make that argument against the weight of societal endorsements of the NGSS content specifications, not to mention the pressure of local political forces.

At the local level, when master K-12 teachers try to alter the NGSS, their course materials, and their class assessment for scientific accuracy and consistency with educational research, they are similarly poised to encounter unwinnable administrative opposition to changes. When held accountable for scores on a "plate-tectonics-free" assessment test derived by the NGSS, will middle school teachers still allocate classroom time teaching their students about the theory of plate tectonics? We would argue that most teachers will not, and those teachers who adhere meticulously to teaching fundamental science concepts may not receive support from administrators who are often well-intentioned but not trained in the science. These administrators are like many in our community: They have relied on the promise of a rigorous and transparent process and are impressed by the endorsements of more than a dozen scientific societies and science education bodies. Against that gravitas, how is a middle school teacher to argue for teaching plate tectonics, the conservation of momentum, or the water cycle?

Given the power of societies' perceived support, they should engage in more rigorous due diligence, and should at a minimum take some simple steps. First, it would be useful for professional societies to provide explicit, detailed commentary on the science in the standards and should do so as quickly as possible. A preliminary fidelity check requires little more than two PDF files, a computer, and perhaps a pot of coffee. From that process, published statements citing specific scientific errors, as well as needed reinsertions and suggestions as to placement and emphasis, can be made. This information can and should be publically available for end-users. Achieve, Inc. has declined to make previous feedback publically available, and the writers from the Frameworks and NGSS have disbanded. Therefore, professional society websites may be the most natural place to distribute these commentaries. We believe that such actions would enhance transparency in the process and that these types of documents would provide much needed guidance for teachers, administrators, schools, professional developers, and publishers.

\section{AUTHOR INFORMATION}

Dr. Stephanie J. Slater is the Director of the CAPER Center for Astronomy \& Physics Education Research, an international collaborative of scholars studying contemporary issues in science education and cognition. Her research focuses on the intersection between learning science and socio-cultural issues. E-mail: stephanie@caperteam.com (Corresponding author)

Dr. Timothy F. Slater holds the University of Wyoming Excellence in Higher Education Chair of Science Education and is a Senior Scientist at the CAPER Center for Astronomy \& Physics Education Research. His research targets effective models for college faculty professional development. E-mail: timslaterwyo@gmail.com

\section{REFERENCES}

Achieve, Inc. (2014). Achieve, Inc. Retrieved from http://www.guidestar.org/organizations/52-2006429/achieve.aspx Alberts, B. (2012). Mobilizing scientific societies. Science, 338(6113), 1396-1396.

American Association for the Advancement of Science. (1993). Benchmarks for science literacy. Oxford University Press.

Coffey, J., \& Alberts, B. (2013). Improving education standards. Science, 339(6119), 489-489. 
Gross, P. R. (2011). Review of the National Research Council's Framework for K-12 Science Education. Thomas B. Fordham Institute.

Krumenaker, L. (2009). The Modern US High School Astronomy Course, Its Status and Makeup II: Additional Results. Astronomy Education Review, 8(1).

[NRC] National Research Council NRC (Ed.). (1996). National science education standards. National Academy Press.

[NRC] National Research Council NRC. (2012). A framework for K-12 science education: Practices, crosscutting concepts, and core ideas. In H. Schweingruber, T. Keller, \& H. Quinn (Eds.). National Academies Press.

[NGSS] Next Generation Science Standards. (2013). Appendix B of Responses to the public drafts. Retrieved from http://www.nextgenscience.org/sites/NGSS/files/Appendix\%20B\%20\%20Responses\%20to\%20the\%20Public\%20Drafts.pdf

O’Donnell, C. L. (2008). Defining, conceptualizing, and measuring fidelity of implementation and its relationship to outcomes in K-12 curriculum intervention research. Review of Educational Research, 78(1), 33-84.

Robelen, E. (2013). Science standards are true to NRC Framework, reviewers conclude. Retrieved May 11, 2015 from http://blogs.edweek.org/edweek/curriculum/2013/04/science_standards_are_true_to_.html 


\section{NOTES}

